\title{
Reflections on Digital Health Tools for Respiratory Applications
}

\author{
Andy Dundon, BSc, ${ }^{1}$ David Cipolla, PhD,,,* Jolyon Mitchell, $\mathrm{PhD},{ }^{3, *}$ and Svetlana Lyapustina, $\mathrm{PhD}^{4, *}$
}

\begin{abstract}
Background: In May 2019, the International Society for Aerosols in Medicine (ISAM) and the International Pharmaceutical Aerosol Consortium on Regulation and Science (IPAC-RS) held a one-day workshop on digital health tools for respiratory therapies, with speakers representing views of patients, pharmaceutical and technology companies, physicians, and payors.

Methods: Workshop presentations, audience discussions, and research of relevant literature form the basis of this article.

Results: Conversations and publications to date illustrate the broad, and growing, interest in digital technologies, but also highlight a few gaps. The key messages are: (1) There are many great technologies, from simple to complex, but there is still an imperfect understanding of the problems that digital tools need to solve. The current approach often starts with a technology "solution" and then identifying the problems that it could address. This is a poor design practice: the offered technology may not represent the optimum solution to that problem; and that "problem" itself may not represent issues that patients or the health care system deem to be of high priority. (2) Respiratory medicine currently lacks widely accepted and easily measured biomarkers or hand-held diagnostic technologies that could be commercialized for complex/heterogeneous diseases such as asthma (unlike in other areas, e.g., diabetes). (3) There are no obvious solutions that integrate the perspectives of the many stakeholdersfrom patients to physicians to payors - to make the new field commercially viable, and the incentives of various parties in the health care system are misaligned. (4) A continued dialog, information sharing, mutual education, and collaborations are important in bringing the promise of digital respiratory medicine to fruition.

Conclusions: Digital tools for diagnosis and management of respiratory conditions are an active area of research and development but long-term success in this field will depend on identifying real needs, and integrating the often-diverging interests of the various partners in each country's health care system.
\end{abstract}

Keywords: digital, insurance, mobile apps, patient-centric, payor

\section{Introduction}

$\mathbf{T}$ he InternationalPharmaceutical Aerosol ConSORTIUM ON REgulation AND SCIENCE (IPAC-RS) ${ }^{(1)}$ and the Standards and Regulatory Network Group of the International Society for Aerosols in Medicine (ISAM) ${ }^{(2)}$ held a workshop "Digital Health in Inhaled Drug Delivery" in May 2019, organized and moderated by the authors of this article. Slides from the workshop are now publicly available $^{(3)}$ for readers interested in specific details presented during that forum. The aim of this report is to summarize discussions and Q\&As at the workshop, and also to provide more recent information in this rapidly maturing field. This report is not intended to represent an official position of either organization.

The workshop's program was designed to elicit views from distinctly different stakeholders in the "health care chain" - spanning those of the patients; health care providers (HCPs) who diagnose and treat those patients; pharmaceutical companies responsible for drug discovery, product

\footnotetext{
${ }^{1}$ Pharmechceutics Ltd., Ware, Hertfordshire, United Kingdom.

${ }^{2}$ Insmed, Inc., Bridgewater, New Jersey.

${ }^{3}$ JMICS, Inc., London, Canada.

${ }^{4}$ Faegre Drinker Biddle \& Reath LLP, Washington, District of Columbia.

*Member of ISAM.
} 
development and commercialization; technology providers who create innovative software and delivery technologies; regulators who decide which products get approved and in some countries how much they will cost; and the payors. The main conclusion is that the health care industry in general, and respiratory treatments in particular, are only at the start of the journey toward integration of digital technologies in a way that would create an ecosystem that would deliver the shared ultimate objective of all partners, which is to improve health outcomes for the patients.

\section{Discussion: Key Messages}

\section{Define unmet needs first}

The opportunity for digital health in the respiratory field is broad. Almost any step on the path from an idea for an intervention to the improved health outcome(s) can be "digitized" in some way. In addition to smart inhalers and acoustic tracking devices, the computer- or softwareenabled applications in the field of respiratory medicine represent a diverse opportunity. Potential consumer segments of digital technologies are numerous-drug product sponsors, academic researchers, community physicians, respiratory technologists, insurance companies, pharmacists, parents of pediatric patients or other family caregivers, and patients themselves. Each group is distinct, with its own needs, capabilities, resources, and requirements.

Many of digital applications already exist or are in development and range across the entire lifecycle of a medical product, including streamlining discovery searches, establishing patterns and mechanisms for disease treatment, automating manufacturing and controls, using smart coldpack sensors for product shipment and storage, monitoring compliance of the patient to their treatment regimen, and supporting patients and their caregivers in various ways. However, key questions addressed in the workshop were whether these solutions are addressing meaningful problems, and whether emerging digital solutions are the best way to address perceived challenges without negatively impacting a partner in the health care ecosystem and creating a potential barrier to adoption.

To identify the proper digital technology solution, one should first, in a structured way, define "the unmet need" as precisely as possible. A product may be successful long term only if it meets a real need. How can the "real needs" be identified?-Start by observing your future users (e.g., be it patients, physicians, manufacturers, etc.), understand their processes, requirements, and preferences; design a few prototypes; then test and evaluate; test again and re-evaluate again; and test and re-evaluate... essentially following the design verification and validation aspects of design control for medical devices. Make no assumptions; test in situations as close to real life as possible. Furthermore, for a pharmaceutical product, this process must be done in the context of the direction of travel of each pharmaceutical company's portfolio. Similar advice was issued by the U.S. Food and Drug Administration (FDA) for devices that collect patients' information-the regulatory agency's guidance for industry ${ }^{(4)}$ recommends that a (biological) question be defined first, along with the target population. Not only design and functionality, but also cybersecurity should start with "understanding patients' lives and perspectives," according to FDA. ${ }^{(5)}$

"Putting patient (or user) at the center" should also recognize there is no single "patient/user." Rather, there are millions of potential users, each with unique needs that, moreover, may change over time. Therefore, the technology needs to be designed so that it can change, too, in order that it can deliver what's best for THIS particular patient, in THIS particular situation. Currently, few applications offer a personalized approach in the management of chronic respiratory conditions ${ }^{(6,7)}$ or consider social and cultural preferences of the target population. ${ }^{(8)}$

As in any technological system, there could be multiple performance targets for the digital solutions, and therefore developing a product or prototype may involve trade-offs. How will the developer decide which performance targets are most important? The answer depends on the goals for the product, or on research questions. ${ }^{(9)}$ Some considerations only come to the surface through real-life testing in real users. For example, in the case of the PRISM initiative (Pediatric Research Using Integrated Sensor Monitoring Systems ${ }^{(10)}$ ), the researchers learned, through trial-and-error and troubleshooting, that lower-cost sensors deployed to users needed more frequent calibrations than the higherquality sensors used in the laboratory. ${ }^{(11,12)}$

Overall, the multiplicity of digital products on offer is a strength of this field, but could become a barrier to widespread adoption if digital products targeting similar areas are not standardized in their features and functionalities, causing user confusion. Another observation arising from the workshop is that functionality of any given application is currently limited.

Admittedly, there are still many "white areas" in the scientific understanding of respiratory diseases, leading to a lack of universally standardized diagnostic or treatment approaches, ${ }^{(13)}$ which in turn deprives technology developers of clear targets against which to design digital tools.

\section{Study, validate, and publish}

There is a paucity of data from appropriately designed ${ }^{(14-16)}$ studies documenting improvements in therapeutic/clinical outcomes, reduced costs, or other promises of digital technology solutions, ${ }^{(17-21)}$ although the number of studies evaluating digital technologies in some form, in therapeutic or lifestyle areas, is growing. ${ }^{(2)}$

Where publications do exist, the underlying data often come from surveys of opinions, feelings, and expectations. Such subjective assessments have some limited value, but cannot substitute for objective measurements. Published studies are often conducted in subjects who are not representative of the target user population, or are biased in methods or analysis. For example, while clinical trial sponsors may feel that a particular technology positively affects patients' experience, those working with patients directly report that some technologies increase the burden on the patients. ${ }^{(23,24)}$ In a number of publications, digital technologies are used as an aid or a tool to explore other research questions (e.g., correlations between air quality and inhaler use in asthmatics)—which, while useful, should not be confused with the study of the digital tools themselves. 
Peer-reviewed publications from well-designed, hypothesisbased studies on digital technologies would go a long way to help convince regulators to approve a new digital product, payors to cover the said product, and physicians and patients to use that product.

\section{Address interoperability}

Interoperability of the devices and platforms needs to be considered early on, as it has the potential to become either a major barrier (if lacking) or a major benefit (if implemented correctly). For example, consider that HCPs have limited time to interact with each patient, and that resources for digital-infrastructure support available to HCPs are also limited. Under these conditions, if different technological solutions used by patients are not interoperable among themselves or with the system(s) used by the HCP, there is a risk that such solutions will not be retained in the long run.

More cautionary lessons could be gleaned from the experience with electronic health records (EHRs) —which could be regarded as a first wave of wide-spread digitalization in health care. EHRs were mandated in the United States by the Patient Protection and Affordable Care Act of 2010, and subsidized through federal tax and other incentives. That mandate led to the emergence of a number of EHR systems lacking interoperability, and though they may have simplified billing in isolated clinical centers, they did not do so across the entire health care system. ${ }^{(25)}$ In retrospect, EHR systems did not reduce overall costs ${ }^{(25)}$ despite stated goals and expectations. Most disappointingly, they have not advanced patient care, ${ }^{(26)}$ and may have even contributed to medical errors. ${ }^{(27)}$ Similar challenges were documented for European health care systems. ${ }^{(28)}$ There is an opportunity, therefore, for organizations involved with the implementation of digital health care solutions to work precompetitively to develop a common digital system architecture, facilitating interoperability.

\section{Who would pay?}

Reimbursement models are at present unclear. Some technology companies have been experimenting with direct deals with select health care service providers, ${ }^{(29)}$ but it is unclear whether those models could be replicated elsewhere, would stand the test of time, and whether they could apply to other types of digital products or even to the same type of product but from different companies. Perhaps digital tools should not rely on traditional health insurance for reimbursement? After all, health insurance companies are just one type-among many - of purchasers of medical products and services. Currently, the financial incentives of the payors, physicians and other HCPs, product manufacturers, "pharmacy benefits managers" (PBM) or their equivalents outside the United States, and patients seem to be all misaligned. A new health care ecosystem is therefore needed.

\section{Regulatory, legal, and practical business constraints}

Technological product developers, especially those with little prior experience in servicing the health care field, too often give too little thought, too late, to the regulatory, legal, and practical business constraints. The goal should be not simply learning about existing regulations. A new mindset is often needed, one that would drive developers to seek out potential risks to the user, proactively, and remove or mitigate them, before the product is brought to the market for the first time.

Developers should be asking themselves: What failure modes are possible, especially in real-life use? How could those failures be detected? How will they be mitigated? If the consequences of "failure" are not life-threatening or even immediately harmful (e.g., a user's annoyance with an overly zealous app, or a user's frustration with a nonresponsive digital service), but if they occur for too many users too many times, the user may be "turned-off" and stop using the app or service. Discontinued use may present not only a business threat but also have a deleterious effect on patients' health outcomes-depending on the app's intended function. For a digital product therefore, the traditional failure modes analysis, which is a key aspect of the design process, should rigorously examine a broader range of potential failure modes, from seemingly minor glitches or insufficient attention to human factors engineering, to electromagnetic interference, mechanical stability, biocompatibility, and other areas relevant to the product's intended use, as well as misuse.

Another aspect of a "regulatory mindset" is that any claim has to be backed up by data-based on real use in real patients with targeted and concomitant health condition(s), not marketing-type surveys. Furthermore, a pharmaceutical sponsor's attention to these matters cannot stop with regulatory approval. Relevant changes in design, sourcing, manufacturing, or quality control methods need to be evaluated and sometimes preapproved; quality needs to be tested on an ongoing basis; and the product's use in the field as well as any user complaints should be monitored, assessed, and addressed (e.g., via a correction, education, or other appropriate response). For products with a digital component, monitoring and timely upgrading based on ever-evolving security threats or to accommodate operating systems updates that are outside of the sponsor's control need to be added to the sponsor's (or vendor's) regulatory and business responsibilities.

From the business perspective, a mismatch in the approaches, terminology, and timelines between traditional pharmaceutical developers and software engineers may contribute to delays and other difficulties, especially if they are not recognized and addressed early on. Iterations and continuous improvements to a health care product are possible, but only if the product is sound enough to be approved for the market in the first place. A plan for validating future changes also needs to be considered ahead of time and cleared with regulators. ${ }^{(30)}$

\section{What regulations apply, anyway?}

Although some uncertainty exists as to specific requirements that regulators may impose on particular products with digital elements, the current environment seems rather welcoming and supportive, ${ }^{(31-34)}$ and FDA even encourages use of "mobile or online technologies to enable virtual or remote follow-up" during clinical investigations ${ }^{(35)}$ of medical devices. Authorities in the United States, ${ }^{(36)}$ Europe ${ }^{(37)}$ and other regions, as well as international standard-setting 
bodies, have already published or are developing requirements that apply to software and related medical products.

According to the most recent explanations from regulators, the extent of their scrutiny-where it's warranted-will depend not only on what the software does (e.g., process physiological signals from sensors) but also on who is the intended user of the software (e.g., HCP vs. the patient or caregiver), as well as other factors (e.g., "state of the health care situation or condition", ( $^{(38)}$

The light-touch approach by FDA and similar agencies, however, while easing the market entry, may be a drawback when negotiating payment models, as insurers prefer to have a regulator's stamp of approval on a medical product before considering reimbursement.

The one area where regulatory scrutiny has been and will continue to be increasing, relates to data privacy ${ }^{39}$ and cybersecurity of digital products. For example, FDA's Center for Devices and Radiological Health $(\mathrm{CDRH})$ includes cybersecurity, along with other aspects of digital health, among its top "Regulatory Science Priorities.", (40,41) Specific requirements will depend on details of the product and on the target market, and will continue to change due to evolving laws, regulations, as well as precedent-setting litigation. Sponsors should therefore procure professional advice for their digital systems as early as possible, on a case-by-case basis.

With respect to privacy, specific laws vary by territory (e.g., the General Data Protection Regulation [GDPR] in Europe and similar laws in several other countries; the Health Insurance Portability and Accountability Act [HIPAA] in the United States; the California Consumer Privacy Act [CCPA] and similar forthcoming acts in other United States; the Personal Information Protection and Electronic Documents Act [PIPEDA] in Canada; and still-evolving laws in China, India, and elsewhere). But the general trend is everincreasing protection of individuals' rights, increasing restrictions on data collection, transfer and use, and increasing penalties for violations of privacy requirements.

\section{Recommendations for Product Developers}

(1) Define the problem to be solved, by observing end users.

(2) Test, and retest the product in users, and optimize the product based on the end users' objective experience with the product, not their expectations, opinions, or projections.

(3) Consider manufacturability at scale. Specifically, for integrated inhalers, manufacturability may impact decisions regarding the energy source, device configuration, materials selection, and other aspects. ${ }^{(42)}$

(4) Take cybersecurity seriously. Find and fix vulnerabilities proactively and make plans to do so on an ongoing basis.

(5) Learn current regulatory requirements for your specific product in the target country, as early in the process as possible. Use that knowledge to steer product design, documentation, and testing; and to inform interactions with investors and business partners.

(6) Consult with payors and explore nontraditional reimbursement models.
(7) Engage in industry-wide discussions to learn and to share best practices. Engage with and understand the perspectives of nonindustry stakeholders. Through broad-based collaborations and collective efforts, help find solutions to common problems.

\section{Conclusions}

There are many players in the health care ecosystemincluding patients, HCPs, regulators, insurance companies and other third-party payors, (bio)pharmaceutical companies, and device/software/technology companies-who all have a shared objective of improving health care outcomes for the patients they serve. However, each of these partners may have different perspectives, barriers, and incentives for how such a system should be designed and the prioritization of problems it must address.

One of the most-repeated comments during the 2019 workshop was that ongoing collaboration among all stakeholders is of paramount importance. Collaborative forums that would allow different parties to dialog and understand each other's perspectives would be valuable, but in themselves are insufficient. More substantive joint work should be undertaken-preferably in ways combining perspectives of various stakeholders - to clarify needs, practices, and expectations; and to the extent possible, develop best-practice guidances and harmonized recommendations to help set directions and priorities for future developments in this field.

\section{Acknowledgments}

The authors thank all of the presenters and attendees of the IPAC-RS/ISAM 2019 workshop.

\section{Author Disclosure Statement}

The authors declare they have no competing financial interests.

\section{Funding Information}

No funding was received for this article.

\section{References}

1. IPAC-RS: Home page. http://ipacrs.org. Accessed February 3, 2020.

2. ISAM: Home page. http://isam.org. Accessed February 3, 2020.

3. IPAC-RS ISAM: Digital health in inhaled drug delivery workshop. 2019. https://ipacrs.org/news-events/events/ digital_health. Accessed February 3, 2020.

4. FDA CDER/CBER: Draft Guidance for Industry. PatientFocused Drug Development: Collecting Comprehensive and Representative Input Guidance for Industry, Food and Drug Administration Staff, and Other Stakeholders. 2018. https://www.fda.gov/media/113653/download. Accessed February 3, 2020.

5. FDA: Executive summary on medical device cybersecurity. 2019. https://www.fda.gov/media/130552/download. Accessed February 3, 2020.

6. Sleurs K, Seys SF, Bousquet J, Fokkens WJ, Gorris S, Pugin P, and Hellings PW: Mobile health tools for the 
management of chronic respiratory diseases. Allergy. 2019; 74:1292-1306.

7. Tendedez H, Ferrario M-A, McNaney R, and Whittle J: Respiratory self-care: identifying current challenges and future potentials for digital technology to support people with chronic respiratory conditions. PervasiveHealth'19: Proceedings of the 13th EAI International Conference on Pervasive Computing Technologies for Healthcare. 2019; 129-138. https://doi.org/10.1145/3329189.3329190. Accessed February 3, 2020.

8. Howard S, Lang A, Sharples S, and Shaw D: See I told you I was taking it!-Attitudes of adolescents with asthma towards a device monitoring their inhaler use: Implications for future design. Appl Ergon. 2017;58:224-237.

9. Williams R, Duvall R, Kilaru V, Hagler G, Hassinger L, Benedict K, Rice J, Kaufman A, Judge R, Pierce G, Allen G, Bergin M, Cohen RC, Fransioli P, Gerboles M, Habre R, Hannigan M, Jack D, Louie P, Martin NA, Penza M, Polidori A, Subramanian R, Ray K, Schauer J, Seto E, Thurston G, Turner J, Wexler AS, and Ning Z: Deliberating performance targets workshop: Potential paths for emerging PM2.5 and O3 air sensor progress. Atmos Environ 2019;2:100031.

10. Sward KA, Bui A, Ambite J-L, and Dellarco M: Pediatric research using integrated sensor monitoring systems (PRISMS): applying sensor technology and informatics to better understand asthma. Proceedings of the 2016 American Medical Informatics Association Annual Symposium. Washington, D.C.; 2016. https://www.mii.ucla.edu/ repository/621.pdf. Accessed February 3, 2020.

11. Erickson B: Predicting asthma attacks in kids. Chem Eng News. 2019;97:18-19.

12. Hosseini A, Buonocore CM, Hashemzadeh S, Hojaiji H, Kalantarian H, Sideris C, Bui AAT, King CE, and Sarrafzadeh M: Feasibility of a secure wireless sensing smartwatch application for the self-management of pediatric asthma. Sensors. 2017;17:1780.

13. Tiotiu A: Biomarkers in asthma: State of the art. Asthma Res Pract. 2018;4:10.

14. Mathews SC, McShea MJ, and Hanley CL: Digital health: A path to validation. NPJ Digit Med. 2019;2:38.

15. Blakey JD, Bender BG, Dima AL, Weinman J, Safioti G, and Costello RW: Digital technologies and adherence in respiratory diseases: The road ahead. Eur Respir J. 2018;52: 1801147.

16. Chen CE, Harrington RA, Desai SA, Mahaffey KW, and Turakhia MP: Characteristics of digital health studies registered in ClinicalTrials.gov. JAMA Intern Med. 2019;179: 838-840.

17. Safavi K, Mathews SC, Bates DW, Dorsey ER, and Cohen AB: Top-funded digital health companies and their impact on high-burden, high-cost conditions. Health Aff (Millwood). 2019;38:1.

18. Adam B: Cohen AB and Safavi K: The oversell and undersell of digital health. Health Affairs Blog. 2019. https:// www.healthaffairs.org/do/10.1377/hblog20190226.63748/ full/. Accessed February 3, 2020.

19. Unni E, Gabriel S, and Ariely R: A review of the use and effectiveness of digital health technologies in patients with asthma. Ann Allergy Asthma Immunol. 2018;121:680-691.

20. Merchant R, Inamdar R, Henderson K, Barrett M, Su JG, Riley J, Van Sickle D, and Stempel D: Digital health intervention for asthma: Patient-reported value and usability. JMIR Mhealth Uhealth. 2018;6:e133.
21. World Health Organization: WHO guideline recommendations on digital interventions for health system strengthening: Research considerations. 2019. https://apps.who.int/iris/ bitstream/handle/10665/311978/WHO-RHR-19.9-eng.pdf? ua=1. Accessed February 3, 2020.

22. Izmailova ES, Wagner JA, and Perakslis ED: Wearable devices in clinical trials: Hype and hypothesis. Clin Pharmacol Ther. 2018;104:42-52.

23. Polhemus AM, Kadhim H, Barnes S, Zebrowski SE, Simmonds A, Masand SN, Banner J, and Dupont M: Accelerating adoption of patient-facing technologies in clinical trials: A pharmaceutical industry perspective on opportunities and challenges. Ther Innov Regul Sci. 2019;53:8-24.

24. Moyer M: Patient technology adoption in clinical trials: 4 barriers holding us back. Clinical Leader. 2019. https:// www.clinicalleader.com/doc/patient-technology-adoptionin-clinical-trials-barriers-holding-us-back-0001. Accessed February 3, 2020.

25. Holland R: Electronic health records were supposed to cut medical costs. They haven't. HBS Working Knowledge. 2018. https://www.forbes.com/sites/hbsworkingknowledge/ 2018/03/07/electronic-health-records-were-supposed-tocut-medical-costs-they-havent/\#6c20d8185060. Accessed February 3, 2020.

26. Schulte F, and Fry E: Death by a thousand clicks. Fortune and KHN. 2019. https://fortune.com/longform/medicalrecords/ and https://khn.org/news/death-by-a-thousandclicks/. Accessed February 3, 2020.

27. Palabindala V, Pamarthy A, and Jonnalagadda NR: Adoption of electronic health records and barriers. J Community Hosp Intern Med Perspect. 2016;6:32643.

28. Baltaxe E, Czypionka T, Kraus M, Reiss M, Askildsen JE, Grenković R, Lindén TS, Pitter JG, Rutten-van Molken M, Solans O, Stokes J, Struckmann V, Roca J, and Cano I: Digital health transformation of integrated care in Europe: Overarching analysis of 17 integrated care programs. J Med Internet Res 2019;21:e14956.

29. Propeller Health: Anthem Blue Cross and Blue Shield collaborates with Propeller Health to support consumers with COPD. https://www.propellerhealth.com/2018/08/16/ anthem-blue-cross-blue-shield-collaborates-propeller-healthsupport-consumers-copd/. Accessed February 3, 2020.

30. FDA: Proposed regulatory framework for modifications to artificial intelligence/machine learning (AI/ML) based software as a medical device (SaMD). 2019. https://www .fda.gov/media/122535/download. Accessed February 3, 2020 .

31. FDA: Digital Health. https://www.fda.gov/medicaldevices/digital-health. Accessed February 3, 2020.

32. Ceretta F: EMA experience with the review of digital technology proposals in medicine development programmes. 3rd Industry Stakeholder Platform on R\&D support. 2018. https://www.ema.europa.eu/en/documents/ presentation/presentation-ema-experience-review-digitaltechnology-proposals-medicine-development-programmes en.pdf. Accessed February 3, 2020.

33. European Commission: eHealth: Digital health and care. https://ec.europa.eu/health/ehealth/overview. Accessed February 3, 2020.

34. Australian Digital Health Agency: Homepage. https://www .digitalhealth.gov.au/. Accessed February 3, 2020.

35. FDA CDRH/CBER: Patient engagement in the design and conduct of medical device clinical investigations. Draft guidance for industry, Food and Drug Administration staff, 
and other stakeholders. 2019. https://www.fda.gov/media/ 130917/download. Accessed February 3, 2020.

36. FDA: Guidances with digital health content. 2019. https:// www.fda.gov/medical-devices/digital-health/guidances-digitalhealth-content. Accessed February 3, 2020.

37. EC, and EP: Regulation (EU) 2017/745 of the European Parliament and of the Council. ("Medical Device Regulation"). Official Journal of the European Union. 2017. https:// eur-lex.europa.eu/eli/reg/2017/745/oj. Accessed February 3, 2020.

38. FDA CDRH, CBER, and CDER: Clinical decision support software. Draft guidance. 2019. https://www.fda.gov/ media/109618/download. Accessed October 2, 2019.

39. Singer N: When apps get your medical data, your privacy may go with it. New York Times. 2019. https://www.nytimes .com/2019/09/03/technology/smartphone-medical-records .html. Accessed February 3, 2020.

40. FDA CDRH: Regulatory science priorities. https://www.fda .gov/medical-devices/science-and-research-medical-devices/ cdrh-regulatory-science-priorities. Accessed February 3, 2020.
41. FDA CDRH: Advisory committee meeting to discuss cybersecurity. 2019. https://www.fda.gov/advisory-committees/ patient-engagement-advisory-committee/september-10-2019patient-engagement-advisory-committee-meeting-announce ment-09102019-09102019. Accessed February 3, 2020.

42. Belton D: Integrating connected health in respiratory: The manufacturing challenge. ONdrugDelivery Magazine. 2018; 89:34-36.

Received on February 10, 2020 in final form, February 11, 2020

Address correspondence to: David Cipolla, PhD Insmed, Inc.

700 US Highway 202/206 Bridgewater, NJ 08807-1704

E-mail: david.cipolla@insmed.com 